\section{Potencial evocado auditivo de tronco encefálico em crianças encaminhadas de um programa de triagem auditiva neonatal}

\section{Brainstem auditive evoked potential in children referred to a neonatal auditive triage program}

Ana Carla Leite Romero 1

Camila Ribas Delecrode 2

Ana Cláudia Viera Cardoso 3

Ana Cláudia Figueiredo Frizzo 4

\begin{abstract}
Objectives: to describe the findings of the brainstem auditive evoked potential (BAEP) test in children on a neonatal auditive triage program and analyze the difference in gender and the interference of age on the measurement of BAEP interwave latency.

Methods: 41 children aged between one and nine months referred to a Municipal Triage Program of the Education and Health Study Centre (EHSS) in the town of Marília, São Paulo State, Brazil in the year of 2010 were evaluated.

Results: a normal result in 31 (75.6\%) and an altered result in $10(24.4 \%)$ patients were observed. Among the latter, alterations principally of the unilateral and bilateral conductive types were observed. It was also observed that with increasing age, the interwave latency decreased in an inverse correlation.

Conclusions: the diagnosis of children in this Neonatal Auditive Triage Program was premature. The results of the $B A E P$ can serve as a reference for other studies in this field. Applying BAEP in this population allows a better direction of conduct and intervention and specific counseling for family members.
\end{abstract}

Key words Hearing, Evoked potentials auditory, Eletrophysiology

\author{
1-4 Programa de Pós-Graduação em Fonoaudiologia. Departamento de \\ Fonoaudiologia. Faculdade de Filosofia e Ciências. Universidade \\ Estadual Paulista. Av. Higyno Muzzi Filho, 737. Campus \\ Universitário. Marília, SP, Brasil. CEP: 17.525-900. \\ E-mail: anafrizzo@marilia.unesp.br
}

\section{Resumo}

Objetivos: descrever os achados do exame de potencial evocado auditivo de tronco encefálico (PEATE) de crianças de um programa de triagem auditiva neonatal e analisar a diferença de gênero e a interferência da idade nas medidas da latência das ondas do PEATE.

Métodos: para tal foram avaliadas 41 crianças com idade entre um a nove meses, referenciadas de um Programa de Triagem Municipal ao Centro de Estudos de Educação e Saúde (CEES) na cidade de Marilia, SP no ano de 2010.

Resultados: foi observado resultado normal em 31 (75,6\%) e alterado em 10 (24,4\%) pacientes. Nesses últimos foram observadas alterações principalmente do tipo condutiva unilateral e bilateral. Observou-se também que a medida que a idade aumenta a latência das ondas diminui numa correlação inversa.

Conclusões: o diagnóstico das crianças deste Programa de Triagem Auditiva Neonatal foi precoce. Os resultados do PEATE poderão servir de referência para outros estudos deste âmbito. O PEATE nesta população permite um melhor direcionamento da conduta e intervenção e aconselhamento específico aos familiares.

Palavras-chave Audição, Potenciais evocados auditivos, Eletrofisiologia 


\section{Introdução}

A integridade do sistema auditivo é condição necessária para a aquisição e o desenvolvimento adequado da linguagem. ${ }^{1}$ É nos primeiros anos de vida que o contato da criança com o meio ambiente garantirá o aprendizado dos sons da fala. ${ }^{2}$

Nos últimos anos, a deficiência auditiva na infância vem sendo discutida no âmbito da saúde pública, visando programar ações em saúde auditiva que permitam prevenir, identificar, diagnosticar e proporcionar a reabilitação auditiva em crianças com deficiência auditiva de origem congênita ou adquirida. ${ }^{3}$

A Triagem Auditiva Neonatal Universal (TANU) foi criada na busca da identificação e intervenção precoce para o desenvolvimento adequado das funções auditivas, desenvolvimento da linguagem e da aprendizagem. ${ }^{4}$ Para a realização da triagem auditiva neonatal a literatura 4 sugere que o protocolo contenha as técnicas de testes combinados de Emissões Otoacústicas (EOA) e Potencial Evocado auditivo de tronco encefálico (PEATE), devido ao fato da primeira avaliar a cóclea, porção mais periférica do sistema auditivo, e a segunda avaliar predominantemente a via auditiva central.

O PEATE tem como objetivos principais identificar alterações no nervo auditivo e tronco encefálico além de estimar o limiar auditivo eletrofisiológico.5,6

Autores relatam que o PEATE sofre influência da maturação auditiva e que os achados deste em crianças nos primeiros meses de vida podem diferir das crianças a partir dos 18 meses. 7,8

$O$ uso do PEATE em recém-nascidos permite observar características diferentes e peculiares das encontradas em crianças acima de dois anos de idade, pois nos recém-nascidos a latência da onda $\mathrm{V}$ diminui devido à mielinização progressiva da via auditiva. 9,10

A maturação do sistema auditivo ocorre em duas fases, primeiramente ocorre na vida intrauterina até por volta do sexto mês de gestação quando há a maturação das vias auditivas periféricas e até os 18 meses de idade quando há a maturação das vias auditivas do sistema nervoso central até o tronco encefálico. 11-14

Um estudo que utilizou o PEATE em recém nascidos relatou que a latência da onda $\mathrm{V}$ diminui devido à mielinização progressiva da via auditiva. ${ }^{9}$ As latências absolutas diminuem com o avanço da idade atingindo a maturidade por volta dos dois meses para a onda I e 18 meses para a onda $\mathrm{V}^{15} \mathrm{em}$ consequência da maturação das vias auditivas do sistema nervoso central até o tronco encefálico durante esta fase do desenvolvimento. 11-14

Durante o primeiro ano de vida os neurônios do tronco encefálico estão amadurecendo e as conexões neurais estão sendo formadas. Neste período o tronco e o tálamo estão começando a conectar-se com o córtex auditivo, quando a entrada sensorial no sistema auditivo é interrompida - em especial durante os primeiros estágios do desenvolvimento as propriedades morfofuncionais dos neurônios no sistema auditivo central podem ser drasticamente alteradas 16 e produzir sequelas irreparáveis para a linguagem e desenvolvimento da fala.

Em decorrência do exposto é fundamental levar em conta a recomendação do Joint Committee on Infant Hearing em inserir na triagem auditiva neonatal universal o PEATE, método capaz de identificar lesões retrococleares e estudar a maturação da via auditiva, quando aplicado na população de recém-nascidos. Esta população apresenta uma maior ocorrência desse tipo de perda, que não pode ser identificada quando se utiliza apenas o registro das EOA. 15,17

A análise dos resultados do PEATE em crianças de um a nove meses de idade poderá servir de referência para outros estudos deste âmbito. $\mathrm{O}$ estudo da maturação da via auditiva a partir dos prontuários permitirá a observação das características peculiares às crianças em fase de desenvolvimento. A compreensão dos achados do PEATE nesta população poderá garantir um melhor direcionamento da conduta e intervenção e aconselhamento aos familiares.

Este estudo teve como objetivo descrever os achados do exame de potencial evocado auditivo de tronco encefálico (PEATE) de crianças de um a nove meses de idade de um programa de triagem auditiva neonatal avaliados num primeiro momento pelas EOA e analisar a diferença de gênero e a interferência da idade nas medidas da latência das ondas do PEATE.

\section{Métodos}

Os dados foram coletados dos prontuários do Setor de Audiologia do Centro de Estudos da Educação e da Saúde (CEES), da Faculdade de Filosofia e Ciências, Universidade Estadual Paulista (UNESP), Campus de Marília, São Paulo.

Esta instituição tem uma parceria com uma maternidade pública municipal para dar suporte ao seu Programa de Triagem Auditiva Neonatal (TAN). Todas as crianças com respostas ausentes $(02 / 04$ bandas de frequência com relação sinal/ruído abaixo de 6 dBNPS) na triagem auditiva utilizando emis- 
sões otoacústicas transientes são referenciados para a Universidade para realização de diagnóstico. Para a avaliação, são utilizados principalmente Potencial Evocado Auditivo de Tronco Encefálico e imitanciometria, o que permite a investigação da audição até seis meses de idade atendendo as recomendações internacionais. 4,18

Foram incluídos no estudo os prontuários de 41 crianças com idade entre um e nove meses, referenciadas para realização de diagnóstico audiológico, atendidas no serviço no ano de 2010. Dos prontuários, foram selecionados os dados de anamnese, potencial evocado auditivo de tronco cerebral e medidas de imitância acústica, quando realizados. Foram excluídos apenas aqueles casos em que os dados eram insuficientes e que por algum motivo não tenha sido possível a realização dos procedimentos supra citados.

No Setor de Audiologia do Centro de Estudos da Educação e da Saúde, UNESP-Marília, o PEATE é realizado com equipamento modelo Eclipse, marca Interacoustics. As respostas foram captadas através de eletrodos posicionados da seguinte maneira: o eletrodo ativo na parte superior da fronte $(\mathrm{Fz})$; mastóides direita (M1) e esquerda (M2) os eletrodos referências e o eletrodo terra lateralmente na fronte (Fpz) de acordo com o padrão Internacional 10-20.19

A estimulação auditiva foi feita por meio do fone de inserção $3 \mathrm{~A}$. Os parâmetros de estimulação foram: estímulo clique, polaridade rarefação, taxa de apresentação de 19,9 cliques/s, sendo promediados de 1000 a 2000 cliques com duplicação de resposta. O filtro passa banda usado foi de $30-3000 \mathrm{~Hz}$ e a janela de análise de $15 \mathrm{~ms}$.

O PEATE é composto por uma série de ondas, denominadas I, III e V, que ocorrem nos primeiros 10 ms após a apresentação do estímulo, captados por eletrodos de superfície posicionados na superfície craniana. Em termos de geradores neurais de resposta dentro do sistema auditivo, o PEATE se origina em vários locais dentro do tronco encefálico. A onda I é resultante da atividade elétrica da porção distal do nervo auditivo. A onda III tem sua origem no núcleo coclear e a onda $\mathrm{V}$ se origina leminisco lateral e colículo inferior. ${ }^{14}$

$\mathrm{Na}$ rotina clínica do Setor de Audiologia do CEES/UNESP-Marília-SP, como protocolo de avaliação do PEATE, inicia-se o exame em 80 decibeis Nível de Audição (dBNA) a fim de verificar a integridade neurofisiológica da via auditiva para análise das latências absolutas das ondas I, III e V e valores dos interpicos I-III; III-V e I-V. Quando se verifica ausência de resposta ou alteração na intensidade a mesma é aumentada gradativamente até 100 dBNA.
Para a pesquisa do nível mínimo as intensidades são progressivamente diminuídas até encontrar a menor intensidade em que a onda $\mathrm{V}$ pode ser observada, sendo fixada em 30 dBNA o nível mínimo a ser pesquisado nas crianças encaminhadas da triagem auditiva.

Os exames foram classificados em normais e alterados: a presença e as latências das ondas I, III e $\mathrm{V}$, interpicos I-III, III-V e I-V dentro dos padrões de normalidade em 80 dBNA (com intervalo entre 1,2 e $7,2 \mathrm{~ms})^{16}$ para a idade cronológica e o limiar eletrofisiológico em 30 dBNA eram indicativos de normalidade. ${ }^{20}$ As alterações foram classificadas em: a) sugestivas de alteração condutiva quando as latências absolutas das ondas I, III e V encontravam-se dentro da normalidade e os valores das latências interpicos I-III, III-V e I-V encontravam-se aumentadas em 80 dBNA e, b) sugestivas de alteração neurossensorial quando os resultados demonstravam ausência de ondas ou presença somente da onda $\mathrm{V}$ em intensidades elevadas, este dado sendo posteriormente confirmado pela imitânciometria. 21

No serviço, em caso de alteração no PEATE é realizada imitanciometria para definir condições de orelha média. A timpanometria é obtida por meio de um analisador de orelha média (Grasson-Standler, modelo GSI-38, com sonda de $226 \mathrm{~Hz}$ ) nas crianças e classificada com base em Jerger. 21

Os resultados do PEATE das crianças com valores dentro dos padrões da normalidade bilateralmente foram agrupados, sendo descritos os valores das latências absolutas e interpicos encontrados em 80 dBNA.

Para verificar a diferença entre gêneros foi utilizado o teste "U" de Mann-Whitney, adotando-se como variáveis dependentes as medidas de latência e interlatência das ondas I, III e V e como variáveis independentes os gêneros masculino e feminino. Para analisar o efeito da idade nas medidas de latência e interlatência das ondas, utilizou-se o coeficiente de correlação de Spearman. O nível de significância foi estabelecido em 0,05 .

O estudo foi autorizado pelo Comitê de Ética e Pesquisa desta instituição, de acordo com o parecer $n^{\circ} 246 / 2011$.

Ao final das avaliações, neste serviço, os pais em geral recebem a orientação por escrito, especialmente em relação à conduta diante de resultados alterados. As crianças com alteração são então contra-referenciadas à Secretaria Municipal de Saúde. Na presença de problema condutivo é sugerida avaliação otorrinolaringológica e quando alterações neurossensoriais são identificadas, as mesmas são encaminhadas para adaptação de 
Aparelho de Amplificação Sonora Individual (AASI) e terapia fonoaudiológica em Serviços de Alta e Média Complexidade da região para seguimento.

\section{Resultados}

Das 41 crianças analisadas, $23(56,1 \%)$ eram do gênero masculino e $18(43,9 \%)$ do feminino, sendo $34(82,9 \%)$ até seis meses de idade e $7(17,1 \%)$ entre sete e nove meses de idade.

Foi observado PEATE normal em 31 (75,6\%) crianças e alterado em $10(24,4 \%)$.

As latências absolutas das ondas e interlatências observadas nos pacientes com resultados nos padrões de normalidade estão descritos na Tabela 1 .

Em relação ao grupo com alteração foram observadas sete sujeitos com alterações do tipo condutiva e três casos com alterações compatíveis com perda auditiva neurossensorial (Figura 1) confirmados pelas timpanometrias do tipo $\mathrm{B}, \mathrm{C}$ e A, respectivamente. Os valores de latência e interlatência do grupo de crianças com resultado alterado são descritos na Tabela 2.

Não houve diferença estatística entre os gêneros em nenhuma das ondas pesquisadas no grupo de pacientes com PEATE normal (Tabela 3).

Com relação à interferência da idade houve relação inversa (Figura 2) no grupo de crianças com PEATE normal conforme a idade aumenta a latência das ondas diminui obtendo-se correlações negativas, na onda III para orelha direita uma correlação de $\mathrm{r}=-0,54(p=0,001)$ enquanto para a onda $\mathrm{V}$ na orelha direita uma correlação $\mathrm{r}=-0,7079(p<0,001)$ e na orelha esquerda $\mathrm{r}=-0,5065(p=0,003)$.

No caso da onda I (OE e OD) e onda III E, não houve correlação significante.

\section{Tabela 1}

Valores de média e desvio-padrão de latência absoluta das crianças com resultados do PEATE dentro dos padrões de normalidade $(\mathrm{N}=31)$.

\begin{tabular}{|c|c|c|c|c|}
\hline \multirow[t]{3}{*}{ Orelha } & & \multicolumn{3}{|c|}{ Latência absolutas e interpicos (ms) } \\
\hline & & $0-3$ meses & $4-6$ meses & 7 - 9 meses \\
\hline & & $\bar{X} \pm D P$ & $\bar{X} \pm D P$ & $\bar{x} \pm D P$ \\
\hline \multirow[t]{6}{*}{ Direita } & Onda I & $1,33 \pm 0,22$ & $1,35 \pm 0,20$ & $1,31 \pm 0,11$ \\
\hline & Onda III & $4,05 \pm 0,26$ & $3,81 \pm 0,20$ & $3,66 \pm 0,24$ \\
\hline & Onda V & $6,30 \pm 0,28$ & $5,92 \pm 0,32$ & $5,64 \pm 0,18$ \\
\hline & Interpico I-III & $2,66 \pm 0,29$ & $2,46 \pm 0,21$ & $2,44 \pm 0,11$ \\
\hline & Interpico III-V & $2,26 \pm 0,24$ & $2,11 \pm 0,21$ & $2,07 \pm 0,38$ \\
\hline & Interpico I-V & $4,98 \pm 0,29$ & $4,25 \pm 0,84$ & $4,32 \pm 0,09$ \\
\hline \multirow[t]{6}{*}{ Esquerda } & Onda I & $1,39 \pm 0,17$ & $1,30 \pm 0,15$ & $1,27 \pm 0,12$ \\
\hline & Onda III & $4,02 \pm 0,26$ & $3,91 \pm 0,35$ & $3,78 \pm 0,12$ \\
\hline & Onda V & $6,26 \pm 0,36$ & $6,08 \pm 0,45$ & $5,65 \pm 0,18$ \\
\hline & Interpico I-III & $2,62 \pm 0,34$ & $2,61 \pm 0,32$ & $2,51 \pm 0,29$ \\
\hline & Interpico III-V & $2,29 \pm 0,25$ & $2,16 \pm 0,29$ & $2,03 \pm 0,31$ \\
\hline & Interpico I-V & $4,87 \pm 0,44$ & $4,78 \pm 0,44$ & $4,38 \pm 0,15$ \\
\hline
\end{tabular}

PEATE = potencial evocado auditivo de tronco encefálico. 
Resultado do PEATE quanto ao tipo de alterações

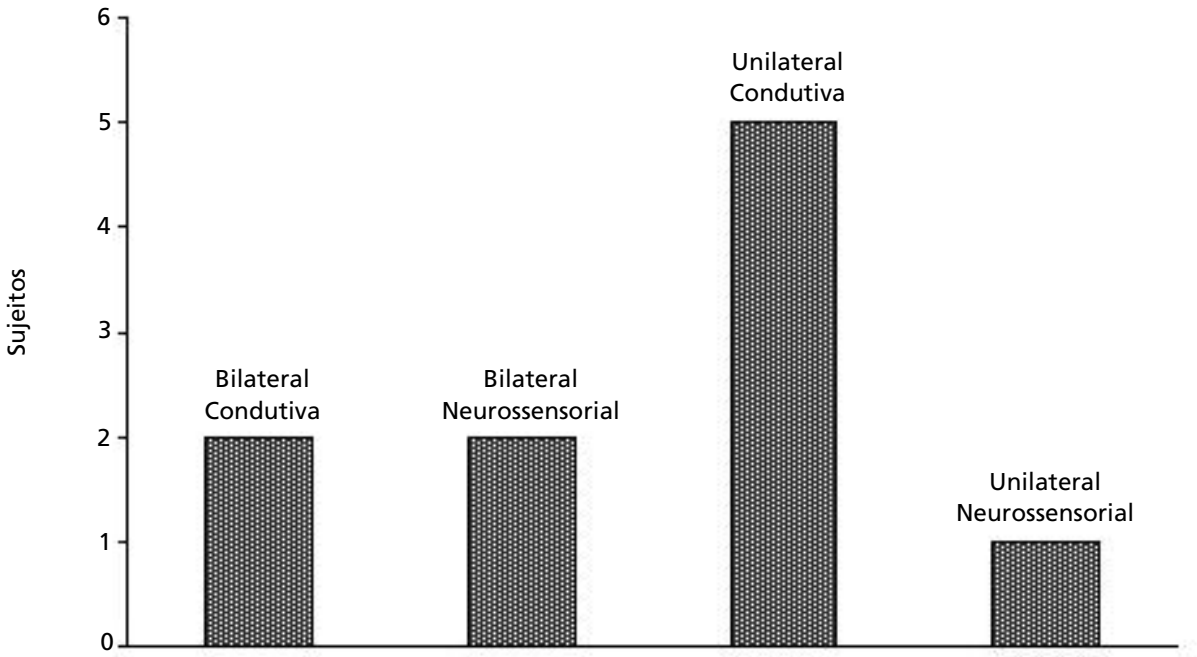

PEATE $=$ potencial evocado auditivo de tronco encefálico. 
Tabela 2

Valores de média e desvio-padrão de latência absoluta e interpicos (ms) das crianças com resultados dos PEATEs alterados $(\mathrm{N}=10)$.

\begin{tabular}{lll}
\hline Orelha & & $\overline{\mathbf{X}} \pm \mathbf{D P}$ \\
\hline \multirow{2}{*}{ Direita } & Onda I & $1,83 \pm 0,52$ \\
& Onda III & $4,16 \pm 0,49$ \\
& Onda V & $6,29 \pm 0,38$ \\
& Interpico I-III & $2,43 \pm 0,06$ \\
& Interpico III-V & $2,08 \pm 0,17$ \\
& Interpico I-V & $4,46 \pm 0,24$ \\
Esquerda & Onda I & $2,00 \pm 0,63$ \\
& Onda III & $4,14 \pm 0,63$ \\
& Onda V & $6,17 \pm 0,51$ \\
& Interpico I-III & $2,48 \pm 0,21$ \\
& Interpico III-V & $2,03 \pm 0,17$ \\
& Interpico I-V & $4,17 \pm 0,25$ \\
\hline
\end{tabular}

PEATE $=$ potencial evocado auditivo de tronco encefálico.

Tabela 3

Efeito do gênero nas latências (ms) das ondas I, III e V nas orelhas direita e esquerda das crianças.

\begin{tabular}{|c|c|c|c|c|}
\hline \multirow{3}{*}{ Ondas no PEATE } & Masculino $(\mathrm{N}=18)$ & Feminino $(\mathrm{N}=13)$ & \multirow{3}{*}{$\underset{\text { ajustado }}{Z}$} & \multirow{3}{*}{$p^{*}$} \\
\hline & & & & \\
\hline & $\bar{X} \pm D P$ & $\bar{X} \pm D P$ & & \\
\hline Onda I OD & $1,32 \pm 0,17$ & $1,32 \pm 0,18$ & $-0,141$ & 0,887 \\
\hline Onda I OE & $1,35 \pm 0,17$ & $1,37 \pm 0,15$ & 0,483 & 0,628 \\
\hline Onda III OD & $3,89 \pm 0,24$ & $4,01 \pm 0,32$ & 1,002 & 0,316 \\
\hline Onda III OE & $3,97 \pm 0,31$ & $3,95 \pm 0,24$ & 0,040 & 0,968 \\
\hline Onda V OD & $6,04 \pm 0,32$ & $6,23 \pm 0,41$ & 1,562 & 0,118 \\
\hline Onda V OE & $6,08 \pm 0,41$ & $6,26 \pm 0,34$ & 0,420 & 0,673 \\
\hline
\end{tabular}

PEATE = potencial evocado auditivo de tronco encefálico; OD = orelha direita; OE = orelha esquerda; * Teste de MannWhitney. 
Interferência da idade na latência (ms) das ondas I, III, e V das orelhas direita e esquerda.

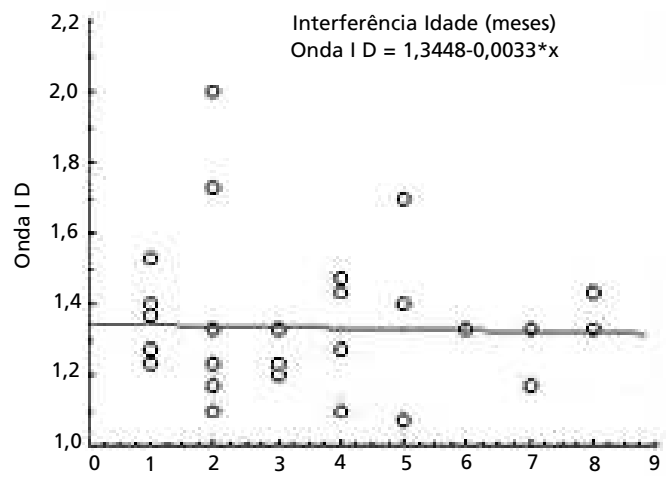

Idade (meses); Onda I D; $r=-0,0342 ; p=0,854$

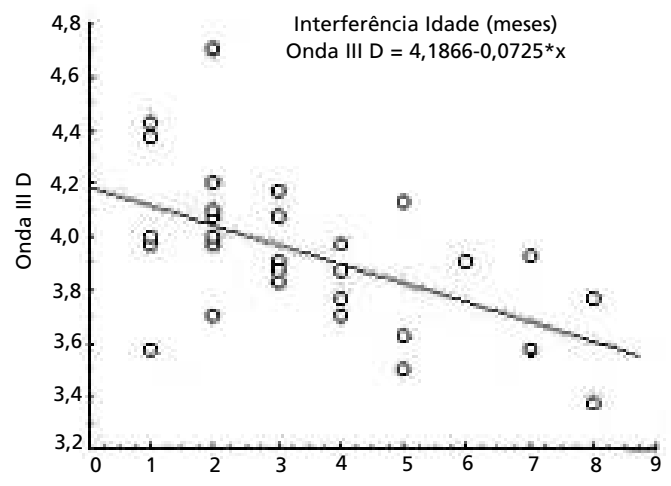

Idade (meses); Onda III D; $r=-0,5400 ; p=0,001$

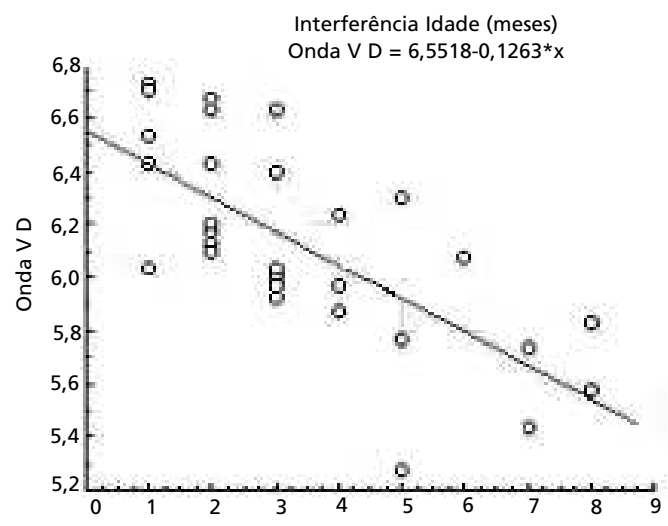

Idade (meses); Onda V D; $r=-0,7079 ; p<0,001$

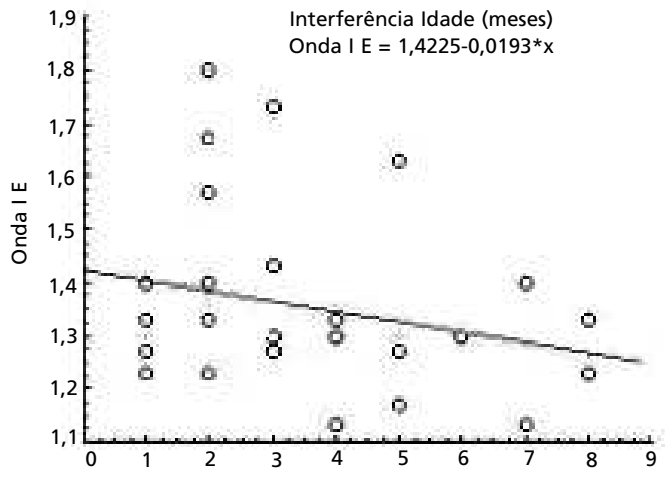

Idade (meses); Onda I E; $\mathrm{r}=-0,2423 ; p=0,189$

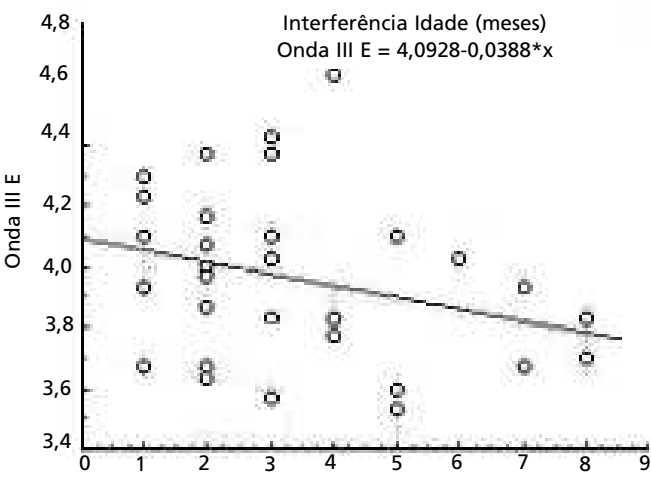

Idade (meses); Onda III $\mathrm{E} ; \mathrm{r}=-0,2895 ; p=0,114$

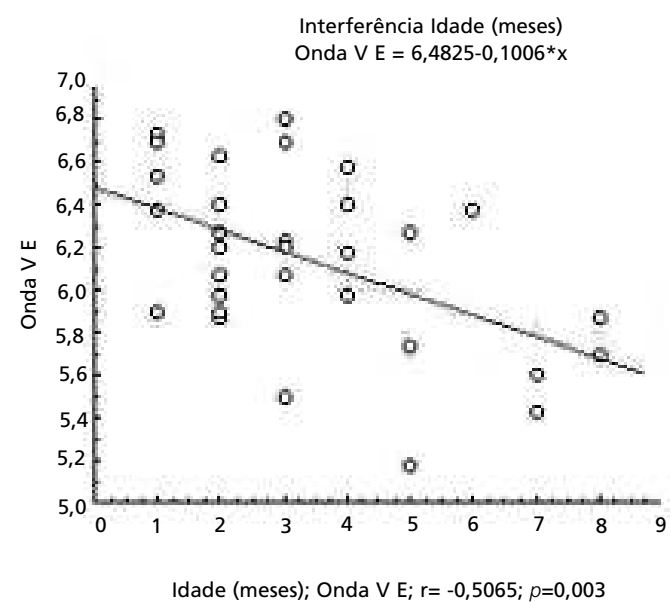

Rev. Bras. Saúde Matern. Infant., Recife, 12 (2): 145-153 abr. / jun., 2012 


\section{Discussão}

Das crianças que falharam na pesquisa das emissões otoacústicas na maternidade, 75,6\% foram identificadas tendo como resultado normal, destacando-se a importância do exame de Potencial Evocado Auditivo de Tronco Encefálico em crianças advindas de programas de triagem auditiva neonatal. $22 \mathrm{O}$ PEATE foi utilizado em recém-nascidos que falharam nas EOA nos Programas de Triagem Auditiva Neonatal em outros estudos, sensibilizando a confirmação da alteração auditiva e garantindo o diagnóstico e o delineamento da intervenção como no caso deste estudo. 23

Em associação com as medidas de imitância acústica o PEATE possibilitou realizar o diagnóstico diferencial entre alterações condutivas e neurosensoriais e estabelecer o limiar eletrofisiológico auditivo.5,6 Quando condutiva, as latências absolutas das ondas I, III e V encontravam-se aumentadas e os valores das latências interpicos I-III, III-V e I-V encontravam-se dentro dos padrões de normalidade em 80 dBNA confirmados pelas timpanometrias dos tipos B e C. No caso das alterações neurossensoriais identificadas aqui, os resultados demonstraram ausência das ondas ou a presença somente da onda $\mathrm{V}$ em intensidades elevadas, associado a timpanometria do tipo A. 21

As crianças que apresentam otites secretoras no período neonatal são de risco para desenvolver otite média no primeiro ano de vida. As alterações de orelha média podem ulteriormente levar a alterações de processamento auditivo e, consequentemente, interferir no desenvolvimento da linguagem e no aprendizado escolar.24

Na comparação das latências absolutas das ondas I, III e V na intensidade de $80 \mathrm{dBNA}$ em relação ao gênero masculino e feminino, não foi encontrada diferença estatística, estando de acordo com a literatura. 25

Os resultados de latência e amplitude das ondas I, III e V e interlatências I-III, III-V e I-V foram condizentes com os valores observados na literatura. ${ }^{23-25} \mathrm{Na}$ comparação entre os valores obtidos para latências absolutas do grupo de crianças com resultados nos padrões de normalidade, com exceção da latência absoluta da onda I, houve aumento da latência em relação aos valores descritos para adultos. 25

\section{Referências}

1. Gatto CL, Tochetto TM. Deficiência auditiva infantil: implicações e soluções. Rev Cefac. 2007; 9: 110-15.
Neste estudo constatamos que a maturação da onda I foi mais rápida, e das ondas III e V, mais lenta confirmando que a maturação da via auditiva até o tronco encefálico segue no sentido caudorostral, sendo a da via periférica mais precoce, e a da rostral, mais tardia podendo seguir até 18 meses de idade. ${ }^{26}$

A influência da idade e da maturação auditiva observada nas crianças de um a nove meses foi significativa. $7,8 \mathrm{O}$ uso do PEATE em recém-nascidos na faixa etária deste estudo exige um cuidado na observação dos valores de latência mais longos das ondas III e $\mathrm{V}$ inerentes à fase maturacional auditiva conforme observado na literatura. 9,10

No presente estudo o valor médio das ondas III e $\mathrm{V}$ das crianças com PEATE normal, atingiu 4,0 ms na onda III e 6,3 ms na onda $\mathrm{V}$, resultados compatíveis com a fase maturacional e a idade cronológica desta população em comparação a outros estudos na mesma faixa etária.9,10,25

Com base neste estudo, sugere-se que o uso do PEATE nesta faixa etária seja analisado criteriosamente evitando-se diagnósticos errôneos. Recomendamos que a sua aplicação em crianças até um ano de idade seja feita considerando os valores de referência da literatura nacional em crianças saudáveis na mesma faixa etária. 25

\section{Considerações finais}

Os achados dos exames de potencial evocado auditivo de tronco encefálico (PEATE) deste programa de triagem auditiva neonatal indicaram que o diagnóstico dessas crianças foi precoce, especialmente quando há associação entre as medidas das emissões otoacústicas, imitância acústica e dos potenciais evocados auditivos. Na comparação em relação ao gênero masculino e feminino, não foi encontrada diferença estatísticamente significante. As medidas de latência das ondas do PEATE tiveram uma correlação inversa com a idade, uma vez que com o passar dos meses foi verificada uma diminuição nos valores de latência. Os resultados do PEATE aqui apresentados podem servir de referência para outros estudos deste âmbito e este instrumento de avaliação mostrou-se útil no monitoramento da maturação auditiva em crianças saudáveis. A compreensão dos achados do PEATE nesta população permite um melhor direcionamento da conduta e intervenção e aconselhamento específico aos familiares.
2. Aurélio FS, Tochetto TM. Triagem auditiva neonatal: experiências de diferentes países. Arq Int Otorrinolaringol. 2010; 14: 355-63. 
3. Alvarenga KF, Bevilacqua MC, Melo TMM, Lopes AC, Moret ALM. Participação das famílias em Programas de Saúde Auditiva: um estudo descritivo. Rev Soc Bras Fonoaudiol. 2011; 16: 49-53.4. American Academy of Pediatrics, Joint Committee on Infant Hearing. Year 2007 position statement: principles and guidelines for early hearing detection and intervention programs. Pediatrics. 2007; 120: 898-921

5. Esteves MCBN, DellAringa AHB, Arruda GV, DellAringa AR, Nardi JC. Estudo das latências das ondas dos potenciais auditivos de tronco encefálico em indivíduos normoouvintes. Braz J Otorhinolaryngol. 2009; 75: 420-5.

6. Jiang ZD, Brosi DM, Li ZH, Chen C, Wilkinson A. Brainstem auditory function at term in preterm babies with and without perinatal complications. Pediatr Res. 2005; 58: 1164-9.

7. Jiang ZD, Wilkson AR. Does peripheral auditory therestold correlat with brainsteam auditory function at term in preterm infants? Acta Oto-laryngol. 2006; 126: 824-27.

8. Karpijoke E, Jaaskelainen S. Neonatal brainstem audiometry with standard neruphisiological auditory evoked potential recording in small premature babies. $8^{\text {th }}$ Congress of International Organization of Societies for eletrophisiological Technology (OSET) proceedings. Am J Electroneurodiagn Technol. 2007; 47: 342-5.

9. Galambos CS, Galambos R. Brainstem evoked response audiometry in newborn hearing screening. Arch Otolaryngol Head Neck Surg. 1979; 105: 86-9.

10. Hecox K, Galambos R. Brainstem auditory evoked responses in humam infant and adults. Arch Otolaryongol. 1974; 99: 30-3.

11. Sleifer P, Costa SS, Cóser PL, Goldani MZ, Dornelles C, Weiss K. Auditory brainstem response in premature and full-term children. Int J Pediatr Otorhinolaryngol. 2007; 71: 449-56.

12. Matas CG. Medidas eletrofisiológicas da audição audiometria de tronco encefálico. In: Carvallo RMM, org. Fonoaudiologia informação para a formação: procedimentos em audiologia. Rio de Janeiro: Guanabara Koogan; 2003. p. 43-57.

13. Hood L. Clinical applications of the auditory brainstem response. San Diego: Singular; 1998.

14. Hall III JW. Handbook of auditory evoked responses. Boston: Allyn and Bacon; 1992.

Recebido em 2 de setembro de 2011

Versão final apresentada em 5 de março de 2012

Aprovado em 10 de abril de 2012
15. Cristobal R, Oghalai JS. Hearing loss in children with very low birth weight: current review of epidemiology and pathophysiology. Arch Dis Child Fetal Neonatal Ed. 2008; 93: 462-8.

16. Sininger YS, Doyle KJ, Moore JK. The case for early identification of hearing loss in children. Auditory system development, experimental auditory deprivation, and development of speech perception and hearing. Pediatr Clin North Am. 1999; 46:1-14.

17. Segre CAM. Prevalência de perda auditiva em recémnascidos de muito baixo peso. J Pediatr. 2003; 79: 103-4.

18. Lewis DR, Marone SAM, Mendes BCA, Cruz OLM, Nóbrega M. Comitê multiprofissional em saúde auditiva: COMUSA. Braz J Otorhinolaryngol. 2010; 76: 121-8.

19. Jasper HH. The ten-twenty electrode system of the international federation. Electroenc Clin Neurophysiol. 1958; 10: $371-5$.

20. Azevedo MF. Emissões otoacústicas. In: Figueiredo MS org. Conhecimentos essenciais para entender bem as emissões otoacústicas e BERA. São José dos Campos: Pulso; 2003. p. 35-83.

21. Jerger J. Clinical experience with impedance audiometry. Arch Otolaryngol. 1970; 92: 311-24.

22. Jardim IS. Emissões otoacústicas evocadas por estímulos transientes e potencial evocado auditivo de tronco encefálico automático na triagem auditiva neonatal [dissertação]. São Paulo: Faculdade de Medicina da Universidade de São Paulo; 2006.

23. Barreira-Nielsen C, Futuro Neto HA, Gattaz G. Processo de implantação de programa de saúde auditiva em duas maternidades públicas. Rev Soc Bras Fonoaudiol. 2007; 12: 99105.

24. Pereira PKS, Azevedo MF, Testa JR. Alterações condutivas em neonatos que falharam na triagem auditiva neonatal. Braz J Otorhinolaryngol. 2010; 76: 347-54.

25. Casali RL, Santos MFC. Potencial evocado auditivo de tronco encefálico: padrão de respostas de lactentes termos e prematuros. Braz J Otorhinolaryngol. 2010; 76: 729-38.

26. Sleifer P. Estudo da maturação das vias auditivas por meio dos potenciais evocados auditivos de tronco encefálico em crianças nascidas pré-termo [dissertação]. Porto Alegre: Universidade Federal do Rio Grande do Sul; 2006. 